\title{
Remarks on certain sums involving floor function
}

\author{
Wachiraya Palatsang ${ }^{\mathrm{a}}$, Prapanpong Pongsriiam ${ }^{\mathrm{b}, *}$, Tammatada Khemaratchatakumthorn ${ }^{\mathrm{b}}$, \\ Kittipong Subwattanachai ${ }^{\mathrm{b}}$, Naruesorn Khuntijit ${ }^{\mathrm{a}}$, Narongkorn Kitnak ${ }^{\mathrm{a}}$ \\ ${ }^{\text {a }}$ Faculty of Education, Silpakorn University, Nakhon Pathom 73000 Thailand \\ b Department of Mathematics, Faculty of Science, Silpakorn University, Nakhon Pathom 73000 Thailand
}

*Corresponding author, e-mail: prapanpong@gmail.com, pongsriiam_p@silpakorn.edu

Received $11 \mathrm{Feb} 2021$

Accepted 9 May 2021

ABSTRACT: For each $a=1,2,3, \ldots, 7$, there exists an integer $b$ depending on $a$ such that

$$
\sum_{k=1}^{n}\left\lfloor\frac{k}{a}\right\rfloor=\left\lfloor\frac{(2 n+b)^{2}}{8 a}\right\rfloor \text { for all } n \in \mathbb{N} .
$$

In this article, we give some remarks on this identity. In particular, we show that the range of $a$ cannot be extended and the value of $b$ is unique.

KEYWORDS: floor function, summation identity, residue class, fractional part

MSC2010: 11A25 11A07 05A19

\section{INTRODUCTION}

Recall that the floor function of a real number $x$, denoted by $\lfloor x\rfloor$, is defined to be the largest integer less than or equal to $x$; and the fractional part of $x$, denoted by $\{x\}$, is defined by $\{x\}=x-\lfloor x\rfloor$. Sums involving the floor function or the fractional part of real numbers have been a popular area of research. For example, in a proof of the quadratic reciprocity law, Gauss shows that for relatively prime positive integers $a, b$,

$$
\sum_{k=1}^{b-1}\left\lfloor\frac{k a}{b}\right\rfloor=\frac{(a-1)(b-1)}{2} .
$$

Dirichlet's divisor problem is to determine the smallest $\theta \geqslant 1 / 4$ such that

$$
\sum_{n \leqslant x}\left\lfloor\frac{x}{n}\right\rfloor=x \log x+(2 \gamma-1) x+O\left(x^{\theta+\varepsilon}\right)
$$

for any $\varepsilon>0$. Hermite's identity states that for $x \in \mathbb{R}$ and $n \in \mathbb{N}$,

$$
\sum_{k=0}^{n-1}\left\lfloor x+\frac{k}{n}\right\rfloor=\lfloor n x\rfloor .
$$

For some recent articles on sums involving the floor function, see for example in the articles by Aursukaree et al [1] for a generalization of Hermite's identity, by Kawsumarng et al $[2,3]$ for the floor function as additive bases, by Onphaeng and Pongsriiam [4] for upper and lower bounds of Jacobsthal-Tverberg sums, by Thanatipanonda and Wong [5] for predictions on sharp bounds for Jacobsthal-Tverberg sums, by Pongsriiam and Vaughan [6] for an improved formula in Dirichlet's divisor problem on arithmetic progressions, Ruankong and Kuhapatanakul [7] for sums involving the floor function and consecutive integral roots, by Phunphayap and Pongsriiam [8] for some applications of the floor function. For more references, see the books by Graham et al [9] and by Pongsriiam [10].

In particular, it is an exercise in Apostol's book [11] to show that for each $a=1,2,3, \ldots, 7$, there exists an integer $b$ depending on $a$ such that

$$
\sum_{k=1}^{n}\left\lfloor\frac{k}{a}\right\rfloor=\left\lfloor\frac{(2 n+b)^{2}}{8 a}\right\rfloor \text { for all } n \in \mathbb{N} .
$$

In this article, we give some remarks on this identity. In particular, we show that a simple formula for $a \geqslant$ 8 does not exist and the value of $b$ for each $a \leqslant 7$ is unique.

\section{PRELIMINARIES AND LEMMAS}

In this section, we give some results which are useful in proving the main theorems. We also give a proof of (1) for completeness. Recall that for $x \in \mathbb{R}$ and $n \in \mathbb{Z}$, we have $\lfloor n+x\rfloor=n+\lfloor x\rfloor$ and $0 \leqslant\{x\}<1$. These are well-known and are often used without 
reference. Next, we prove a lemma that are applied throughout this article.

Lemma 1 Let $n \geqslant 0, a \geqslant 1,0 \leqslant r<a$ be integers, and let $n \equiv r(\bmod a)$. Suppose $b=2-a$. Then

$$
\sum_{k=1}^{n}\left\lfloor\frac{k}{a}\right\rfloor=\frac{n(n+b)-r(b+r)}{2 a} .
$$

Proof: If $a=1$, then $r=0, b=1$, and

$$
\sum_{k=1}^{n}\left\lfloor\frac{k}{a}\right\rfloor=\sum_{k=1}^{n} k=\frac{n(n+1)}{2}=\frac{n(n+b)-r(b+r)}{2 a} .
$$

If $n<a$, then $r=n$ and both sides of (2) are zero. So we assume that $n \geqslant a \geqslant 2$. Since $n \equiv r(\bmod a)$ and $n \geqslant a>r$, there exists $q \in \mathbb{Z}^{+}$such that $n=a q+r$. Therefore the left-hand side of (2) can be written as

$$
\sum_{k=0}^{a q-1}\left\lfloor\frac{k}{a}\right\rfloor+\sum_{k=a q}^{a q+r}\left\lfloor\frac{k}{a}\right\rfloor
$$

If $a q \leqslant k \leqslant a q+r$, then $\lfloor k / a\rfloor=q$, and so the second sum above is equal to $q(r+1)$. The first sum can be written as

$$
\sum_{0 \leqslant \ell<q}\left(\sum_{a \ell \leqslant k<a(\ell+1)}\left\lfloor\frac{k}{a}\right\rfloor\right)=\sum_{0 \leqslant \ell<q} a \ell=\frac{a q(q-1)}{2} .
$$

Combining the first and second sums and substituting $q=\frac{n-r}{a}$, we see that the left-hand side of (2) is equal to

$$
\begin{aligned}
\frac{1}{2} & \left(a q^{2}-a q+2 q r+2 q\right) \\
& =\frac{1}{2 a}\left(a^{2}\left(\frac{n-r}{a}\right)^{2}-a^{2}\left(\frac{n-r}{a}\right)+2(n-r) r+2(n-r)\right) \\
& =\frac{1}{2 a}\left(n^{2}-2 n r+r^{2}-a n+a r+2 n r-2 r^{2}+2 n-2 r\right) \\
& =\frac{1}{2 a}(n(n+2-a)-r(r+2-a)),
\end{aligned}
$$

which is equal to the right-hand side of (2).

By applying Lemma 1, we can prove (1) conveniently as shown in the next theorem.

Theorem 1 If $a \leqslant 7$ is a positive integer, then we can choose $b=2-a$ so that

$$
\sum_{k=1}^{n}\left\lfloor\frac{k}{a}\right\rfloor=\left\lfloor\frac{(2 n+b)^{2}}{8 a}\right\rfloor \text { for all } n \in \mathbb{N} .
$$

Proof: We first consider the case $a=1$. Then the left-hand side of (3) is $n(n+1) / 2$ while the righthand side of (3) is equal to

$$
\left\lfloor\frac{(2 n+1)^{2}}{8}\right\rfloor=\left\lfloor\frac{n(n+1)}{2}+\frac{1}{8}\right\rfloor=\frac{n(n+1)}{2},
$$

where the last equality is obtained from the fact that $n(n+1) / 2$ is an integer. The proofs for $a=2$ to $a=7$ are similar, so we show the details only in the cases $a=6$ and $a=7$. So suppose that $a=6$. By Lemma 1, we obtain

$$
\sum_{k=1}^{n}\left\lfloor\frac{k}{6}\right\rfloor= \begin{cases}\frac{n(n-4)}{12}, & \text { if } n \equiv 0,4 \quad(\bmod 6) ; \\ \frac{n(n-4)+3}{12}, & \text { if } n \equiv 1,3 \quad(\bmod 6) ; \\ \frac{n(n-4)+4}{12}, & \text { if } n \equiv 2 \quad(\bmod 6) ; \\ \frac{n(n-4)-5}{12}, & \text { if } n \equiv 5 \quad(\bmod 6) .\end{cases}
$$

The right-hand side of (3) is equal to

$\left\lfloor\frac{(2 n-4)^{2}}{48}\right\rfloor=\left\lfloor\frac{4 n^{2}-16 n+16}{48}\right\rfloor=\left\lfloor\frac{n(n-4)}{12}+\frac{16}{48}\right\rfloor$.

If $n \equiv 0,4,6,10(\bmod 12)$, then $n(n-4) \equiv 0$ $(\bmod 12)$, and so

$$
\left\lfloor\frac{n(n-4)}{12}+\frac{16}{48}\right\rfloor=\frac{n(n-4)}{12} .
$$

If $n \equiv 1,3,7,9(\bmod 12)$, then $n(n-4) \equiv-3$ $(\bmod 12)$, and therefore

$\left\lfloor\frac{n(n-4)}{12}+\frac{16}{48}\right\rfloor=\left\lfloor\frac{n(n-4)+3}{12}+\frac{4}{48}\right\rfloor=\frac{n(n-4)+3}{12}$.

If $n \equiv 2,8(\bmod 12)$, then $n(n-4) \equiv-4(\bmod 12)$, and thus

$$
\left\lfloor\frac{n(n-4)}{12}+\frac{16}{48}\right\rfloor=\left\lfloor\frac{n(n-4)+4}{12}\right\rfloor=\frac{n(n-4)+4}{12} .
$$

If $n \equiv 5,11(\bmod 12)$, then $n(n-4) \equiv 5(\bmod 12)$ and hence

$\left\lfloor\frac{n(n-4)}{12}+\frac{16}{48}\right\rfloor=\left\lfloor\frac{n(n-4)-5}{12}+\frac{36}{48}\right\rfloor=\frac{n(n-4)-5}{12}$.

From these, we obtain

$$
\begin{aligned}
& \left\lfloor\frac{(2 n+b)^{2}}{8 a}\right\rfloor=\left\lfloor\frac{n(n-4)}{12}+\frac{16}{48}\right\rfloor \\
& \quad= \begin{cases}\frac{n(n-4)}{12}, & \text { if } n \equiv 0,4,6,10 \quad(\bmod 12) ; \\
\frac{n(n-4)+3}{12}, & \text { if } n \equiv 1,3,7,9(\bmod 12) ; \\
\frac{n(n-4)+4}{12}, & \text { if } n \equiv 2,8(\bmod 12) ; \\
\frac{n(n-4)-5}{12}, & \text { if } n \equiv 5,11 \quad(\bmod 12) .\end{cases}
\end{aligned}
$$

Observe that $n \equiv 0,4(\bmod 6)$ if and only if $n \equiv$ $0,4,6,10(\bmod 12) ; n \equiv 1,3(\bmod 6)$ if and only if $n \equiv 1,3,7,9(\bmod 12) ; n \equiv 2(\bmod 6)$ if and only if $n \equiv 2,8(\bmod 12) ; n \equiv 5(\bmod 6)$ if and only if $n \equiv 5,11(\bmod 12)$. Comparing (4) and (5), we see that

$$
\sum_{k=1}^{n}\left\lfloor\frac{k}{6}\right\rfloor=\left\lfloor\frac{(2 n+b)^{2}}{8 a}\right\rfloor .
$$


So this theorem is proved for $a=6$. Next, consider $a=7$. By Lemma 1 , we have

$$
\sum_{k=1}^{n}\left\lfloor\frac{k}{7}\right\rfloor= \begin{cases}\frac{n(n-5)}{14}, & \text { if } n \equiv 0,5 \quad(\bmod 7) ; \\ \frac{n(n-5)+4}{14}, & \text { if } n \equiv 1,4 \quad(\bmod 7) ; \\ \frac{n(n-5)+6}{14}, & \text { if } n \equiv 2,3 \quad(\bmod 7) ; \\ \frac{n(n-5)-6}{14}, & \text { if } n \equiv 6 \quad(\bmod 7) .\end{cases}
$$

The right-hand side of (3) is equal to

$$
\left\lfloor\frac{(2 n-5)^{2}}{56}\right\rfloor=\left\lfloor\frac{4 n^{2}-20 n+25}{56}\right\rfloor=\left\lfloor\frac{n(n-5)}{14}+\frac{25}{56}\right\rfloor \text {. }
$$

Similar to the case $a=6$, we calculate $n(n-5) \bmod$ 14 according to the residues of $n$ modulo 14 and obtain that

$$
\begin{aligned}
& \left\lfloor\frac{n(n-5)}{14}+\frac{25}{56}\right\rfloor \\
& \quad= \begin{cases}\frac{n(n-5)}{14}, & \text { if } n \equiv 0,5,7,12 \quad(\bmod 14) ; \\
\frac{n(n-5)+4}{14}, & \text { if } n \equiv 1,4,8,11 \quad(\bmod 14) ; \\
\frac{n(n-5)-6}{14}, & \text { if } n \equiv 2,3,9,10 \quad(\bmod 14) ;\end{cases}
\end{aligned}
$$

Comparing (6) and (7), we see that this theorem is verified for $a=7$. Hence the proof is complete.

\section{MAIN RESULTS}

In this section, we show that $b$ in Theorem 1 , after a reduction, is necessarily equal to $2-a$ and the range of $a \leqslant 7$ cannot be extended to any positive integer larger than 7 .

Theorem 2 Let $a \in \mathbb{N}, b, c, d \in \mathbb{R}$, and $d \neq 0$. Suppose that $A \subseteq \mathbb{N}$ is an infinite set and

$$
\sum_{k=1}^{n}\left\lfloor\frac{k}{a}\right\rfloor=\left\lfloor\frac{(c n+b)^{2}}{d a}\right\rfloor \text { for all } n \in A .
$$

Then

$$
\frac{(c n+b)^{2}}{d a}=\frac{(2 n+2-a)^{2}}{8 a} \text { for all } n \in A .
$$

Proof: Let $n \in A$. By Lemma 1, the left-hand side of (8) is equal to

$\frac{n(n+2-a)-r(2-a+r)}{2 a}=\frac{n^{2}}{2 a}+\frac{n(2-a)}{2 a}-\frac{r(2-a+r)}{2 a}$,

where $0 \leqslant r<a$ and $n \equiv r(\bmod a)$. Recall that $\lfloor x\rfloor=x-\{x\}$ and $0 \leqslant\{x\}<1$. So the right-hand side of (8) can be written as

$$
\frac{n^{2} c^{2}}{d a}+\frac{2 n b c}{d a}+\frac{b^{2}}{d a}-f_{1}(n, a, b, c, d),
$$

where $0 \leqslant f_{1}(n, a, b, c, d)<1$. Dividing both sides of (8) by $n^{2}$, we obtain

$$
\begin{aligned}
\frac{1}{2 a}+ & \frac{2-a}{2 a n}-\frac{r(2-a+r)}{2 a n^{2}} \\
& =\frac{c^{2}}{d a}+\frac{2 b c}{d a n}+\frac{b^{2}}{d a n^{2}}-\frac{f_{1}(n, a, b, c, d)}{n^{2}} .
\end{aligned}
$$

Since (9) holds for all $n \in A$, we can take limit as $n \in A$ and $n \rightarrow \infty$ on both sides of (9) which leads to $\frac{1}{2 a}=\frac{c^{2}}{d a}$. Therefore $c^{2}=\frac{d}{2}$ and (9) reduces to

$$
\begin{aligned}
\frac{2-a}{2 a n}- & \frac{r(2-a+r)}{2 a n^{2}} \\
& =\frac{2 b c}{d a n}+\frac{b^{2}}{d a n^{2}}-\frac{f_{1}(n, a, b, c, d)}{n^{2}} .
\end{aligned}
$$

Multiplying both sides of (10) by $n$ and taking limit as $n \in A$ and $n \rightarrow \infty$, we obtain $\frac{2-a}{2 a}=\frac{2 b c}{d a}$. Then

$$
\frac{(2-a) c}{2}=\frac{2 b c^{2}}{d}=b \text {. }
$$

From these, we obtain

$$
\begin{aligned}
\frac{(c n+b)^{2}}{d a} & =\frac{c^{2} n^{2}}{d a}+\frac{2 b c n}{d a}+\frac{b^{2}}{d a} \\
& =\frac{n^{2}}{2 a}+\frac{(2-a) n}{2 a}+\frac{(2-a)^{2} c^{2}}{4 d a} \\
& =\frac{4 n^{2}}{8 a}+\frac{4(2-a) n}{8 a}+\frac{(2-a)^{2}}{8 a} \\
& =\frac{(2 n+2-a)^{2}}{8 a} .
\end{aligned}
$$

This completes the proof.

Theorem 2 immediately implies that it is necessary to choose $b=2-a$ in Theorem 1 .

Corollary 1 The value $b=2-a$ in Theorem 1 is unique. That is, if $b \in \mathbb{R}, a \in \mathbb{N}, a \leqslant 7$, and

$\sum_{k=1}^{n}\left\lfloor\frac{k}{a}\right\rfloor=\left\lfloor\frac{(2 n+b)^{2}}{8 a}\right\rfloor$ for infinitely many $n \in \mathbb{N}$, then $b=2-a$.

Proof: By Theorem 2, we have

$$
\frac{(2 n+b)^{2}}{8 a}=\frac{(2 n+2-a)^{2}}{8 a} .
$$

Since (11) holds for infinitely many $n \in \mathbb{N}$, we can choose distinct positive integers $n_{0}$ and $n_{1}$ and substitute $n=n_{0}$ and $n=n_{1}$ in (11) to obtain

$$
\begin{aligned}
& 4 n_{0} b+b^{2}=4 n_{0}(2-a)+(2-a)^{2}, \\
& 4 n_{1} b+b^{2}=4 n_{1}(2-a)+(2-a)^{2} .
\end{aligned}
$$


Subtracting (13)-(12), we obtain $b=2-a$, as desired.

Next, we show that the range of $a \leqslant 7$ in Theorem 1 cannot be extended.

Theorem 3 For each positive integer $a \geqslant 8$ and for any choice of $b, c, d \in \mathbb{R}$ with $d \neq 0$, there are infinitely many $n \in \mathbb{N}$ such that

$$
\sum_{k=1}^{n}\left\lfloor\frac{k}{a}\right\rfloor \neq\left\lfloor\frac{(c n+b)^{2}}{d a}\right\rfloor .
$$

Proof: Suppose for a contradiction that there exist $a \in \mathbb{N}$ and $b, c, d \in \mathbb{R}$ such that $a \geqslant 8, d \neq 0$, and

$$
\sum_{k=1}^{n}\left\lfloor\frac{k}{a}\right\rfloor \neq\left\lfloor\frac{(c n+b)^{2}}{d a}\right\rfloor
$$

for only a finite number of $n \in \mathbb{N}$. Then there exists $M \in \mathbb{N}$ such that

$$
\sum_{k=1}^{n}\left\lfloor\frac{k}{a}\right\rfloor=\left\lfloor\frac{(c n+b)^{2}}{d a}\right\rfloor \text { for all } n \geqslant M .
$$

By Theorem 2, we have

$$
\sum_{k=1}^{n}\left\lfloor\frac{k}{a}\right\rfloor=\left\lfloor\frac{(2 n+2-a)^{2}}{8 a}\right\rfloor \text { for all } n \geqslant M .
$$

Let $n \geqslant M$ and $n \equiv a-1(\bmod 2 a)$.

Then $n(n+2-a) \equiv a-1(\bmod 2 a)$ and $n \equiv a-1$ $(\bmod a)$. By Lemma 1 , we obtain

$$
\sum_{k=1}^{n}\left\lfloor\frac{k}{a}\right\rfloor=\frac{n(n+2-a)-(a-1)}{2 a} \in \mathbb{Z} .
$$

Next, we calculate the right-hand side of (14). We have

$$
\begin{aligned}
& \left\lfloor\frac{(2 n+2-a)^{2}}{8 a}\right\rfloor \\
& \quad=\left\lfloor\frac{n(n+2-a)-(a-1)}{2 a}+\frac{a-1}{2 a}+\frac{(2-a)^{2}}{8 a}\right\rfloor \\
& \quad=\frac{n(n+2-a)-(a-1)}{2 a}+\left\lfloor\frac{a-1}{2 a}+\frac{(2-a)^{2}}{8 a}\right\rfloor .
\end{aligned}
$$

But $\frac{a-1}{2 a}+\frac{(2-a)^{2}}{8 a}=\frac{a}{8} \geqslant 1$, and so

$$
\left\lfloor\frac{a-1}{2 a}+\frac{(2-a)^{2}}{8 a}\right\rfloor \geqslant 1 .
$$

By (15), (16), and (17), we obtain

$$
\sum_{k=1}^{n}\left\lfloor\frac{k}{a}\right\rfloor<\left\lfloor\frac{(2 n+2-a)^{2}}{8 a}\right\rfloor,
$$

which contradicts (14). Hence the proof is complete.
Remark 1 Obviously, the sum $\sum_{k=1}^{n}\left\lfloor\frac{k}{a}\right\rfloor$ depends on $a$ and $n$. If $a=1,2,3, \ldots, 7$, then Theorem 1 simply says that a simple formula for this sum exists; but if $a$ is a positive integer larger than 7 , then Theorem 3 states that such a simple formula does not exist. Nevertheless, we can always use Lemma 1 to evaluate this sum though it may lead to many cases of residues modulo $a$ as shown in the following example.

Example 1 If $a=8$, we can apply Lemma 1 to obtain

$$
\sum_{k=1}^{n}\left\lfloor\frac{k}{8}\right\rfloor= \begin{cases}\frac{n(n-6)}{16}, & \text { if } n \equiv 0,6 \quad(\bmod 8) ; \\ \frac{n(n-6)+5}{16}, & \text { if } n \equiv 1,5 \quad(\bmod 8) ; \\ \frac{n(n-6)+8}{16}, & \text { if } n \equiv 2,4 \quad(\bmod 8) ; \\ \frac{n(n-6)+9}{16}, & \text { if } n \equiv 3 \quad(\bmod 8) ; \\ \frac{n(n-6)-7}{16}, & \text { if } n \equiv 7 \quad(\bmod 8) .\end{cases}
$$

Questions: We have obtained the results for all positive integers $a$. Can we extend them to negative integers? What about nonzero rational numbers? Can we say something nontrivial about the sum $\sum_{k=1}^{n}\left\lfloor\frac{k}{a}\right\rfloor$ when $a$ is positive irrational? What happen if we replace the floor by the ceiling function? We leave these questions to the interested readers.

Acknowledgements: We thank the reviewers for their comments and suggestions which improve the quality of this paper. Prapanpong Pongsriiam is jointly supported by the Faculty of Science Silpakorn University and the National Research Council of Thailand, grant number NRCT5-RSA63021-02. Kittipong Subwattanachai has received a DPST scholarship of IPST, Thailand.

\section{REFERENCES}

1. Aursukaree S, Khemaratchatakumthorn T, Pongsriiam P (2019) Generalizations of Hermite's identity and applications. Fibonacci Q 57, 126-133.

2. Kawsumarng $S$, Khemaratchatakumthorn T, Noppakaew P, Pongsriiam P (2021) Sumsets associated with Wythoff sequences and Fibonacci numbers. Period Math Hung 82, 98-113.

3. Kawsumarng S, Khemaratchatakumthorn T, Noppakaew P, Pongsriiam P (2020) Distribution of Wythoff sequences modulo one. Int J Math Comput Sci 15, 1045-1053.

4. Onphaeng K, Pongsriiam P (2017) Jacobsthal and Jacobsthal-Lucas numbers and sums introduced by Jacobsthal and Tverberg. J Integer Seq 20, ID 17.3.6.

5. Thanatipanonda T, Wong E (2018) Curious bounds for floor function sums. J Integer Seq 21, ID 18.1.8.

6. Pongsriiam P, Vaughan RC (2015) The divisor function on residue classes I. Acta Arithmetica 168, 369-381. 
7. Ruankong P, Kuhapatanakul K (2019) Approximating sums of consecutive integral roots. J Integer Seq 22, ID 19.6.8.

8. Phunphayap P, Pongsriiam P (2020) Explicit formulas for the $p$-adic valuations of Fibonomial coefficients II. AIMS Math 5, 5685-5699.
9. Graham RL, Knuth DE, Patashnik O (1994) Concrete Mathematics 2nd edn, Addison-Wesley.

10. Pongsriiam P (2020) Introduction to Analytic Number Theory, Silpakorn University Printing House, Thailand.

11. Apostol TM (1976) Introduction to Analytic Number Theory, Springer. 